\title{
On dusty gas model governed by the Kuramoto-Sivashinsky equation
}

\author{
GLEB G. DORONIN* and NIKOLAI A. LARKIN ${ }^{\dagger}$ \\ Departamento de Matemática, Universidade Estadual de Maringá, 87020-900, Maringá, Brazil \\ E-mail: ggdoronin@uem.br/nlarkine@uem.br
}

\begin{abstract}
Initial and initial-boundary value problems for the Kuramoto-Sivashinsky model of "gas - solid particles" media are considered. Existence, uniqueness and exponential decay of global strong solutions are proved for small initial data.
\end{abstract}

Mathematical subject classification: 35M20, 35Q72.

Key words: dusty gas, nonlinear problems, global solvability, stability.

\section{Introduction}

There are a number of mathematical models describing nonstationary two-phase motions of gas-particles mixtures known as "dusty gases" [1]. These models are usually based either on the Navier-Stokes equations [1,2], or exploit inviscid gasdynamics approach [3-5]. There are models that include viscous equations for a carrier gas and first-order hyperbolic equations for particles [6-10].

Interest in dusty gases dates from the 1930's due to necessity to simulate processes of the solid fuel combustion in rockets nozzles. Probably, one of the first mathematical works in this field is Reference [4]. The main feature of the corresponding system of PDE's is that it is not hyperbolic. This circumstance made the mathematical modeling doubtful and numerical algorithms unstable. To improve stability of numerical schemes, it was suggested to consider more regular initial data [19]. However, this approach does not lead to the wellposedness. Indeed, it was shown in [9] that the Cauchy problem for this model

\#562/03. Received: 04/II/03. Accepted: 18/III/04.

*Partially supported by CNPq-Brazil, Grant 300544/00-6.

${ }^{\dagger}$ Partially supported by CNPq-Brazil, Grant 300462/98-0. 
does not possess even local-in-time solutions within classes of functions with a finite number of derivatives. Only analytic solutions do exist due to the CauchyKovalevsky theorem. This phenomenon has the same nature as the Cauchy problem for the Laplace equation. On the other hand, considering equations with dissipative terms, one may expect more regular properties of solutions.

If to use for the carrier phase the Navier-Stokes equations (second order dissipation), then it is possible to prove local-in-time solvability of the Cauchy or mixed problems [8-10]. However, when the phase interaction term contains the local concentration of a dispersive phase, we do not know any results on the existence of global in $t$ solutions. The first step towards the global solvability was made in [7]: the local concentration of particles was substituted by the averaged constant concentration that made it possible to prove the existence of global weak solutions to the mixed problem.

In this paper we propose to model the carrier phase of a dusty gas by the Kuramoto-Sivashinsky equation which has the dissipative term of the fourth order and is widely used in the theory of viscous turbulent flows and in studies of flame fronts propagation [11-15]. The last ones provide classical examples of dusty media. This approach is a development and generalization of our previous results [18]. Instead of an averaged concentration of particles in the phase interaction term we use a local dust concentration. This provides more technical and ideological difficulties. Our goal is to investigate the well-posedness of the mixed and Cauchy problems for this model. We prove the existence and uniqueness of global strong solutions for small initial data. The method of successive approximations, compactness arguments and the continuation of a local solution are used.

If the total mass of solid particles is sufficiently small, then the exponential decay of solutions is proved. This means an asymptotic stability of a steady state of a dusty gas flow.

It should be noted that offered proofs are of constructive character that may serve as a basis for stable and rapid numerical algorithms. 


\section{Main results}

For $T>0$, let $Q=\{(x, t): x \in \Omega, t \in(0, T)\}$ where $\Omega \subseteq \mathbb{R}$ is either the interval $\Omega=(0,1)$ in the case of mixed problem or the line $\Omega=\mathbb{R}$ in the case of the Cauchy problem. In $Q$ we consider the following problem:

$$
\begin{aligned}
& u_{t}+u u_{x}+\mu u_{x x}+v u_{x x x x}+\alpha u=m K(v-u), \\
& v_{t}+v v_{x}=K(u-v), \\
& m_{t}+(m v)_{x}=0 \text {, } \\
& u(x, 0)=u_{0}(x), \quad v(x, 0)=v_{0}(x), \quad m(x, 0)=m_{0}(x) \geq 0, \\
& \left.\begin{array}{l}
u(0, t)=u_{x x}(0, t)=u(1, t)=u_{x x}(1, t)=0, \\
v(0, t)=v(1, t)=0,
\end{array}\right\} \text { if } \Omega=(0,1) .
\end{aligned}
$$

Here $u$ and $v$ are velocities of the medium and solid particles respectively; $m$ is the local concentration of particles; $\mu, v$ and $\alpha$ are positive constant coefficients of viscosity and friction and $K>0$ is the constant coefficient of the phase interaction.

Remark 1. It can be seen from (2.5) that $x=0$ and $x=1$ are characteristics of hyperbolic equations (2.2) and (2.3). This justifies the formulation of the mixed problem (2.1)-(2.5).

To formulate main results we define a real $\lambda$ as follows:

$$
\begin{aligned}
\lambda= & {\left[\left\|v_{0}^{\prime}\right\|^{2}+\frac{K\left(\left\|u_{0}\right\|^{2}+\left\|\sqrt{m_{0}} v_{0}\right\|^{2}\right)}{2 \alpha}+\frac{K\left(\left\|u_{0}\right\|^{2}+\left\|\sqrt{m_{0}} v_{0}\right\|^{2}\right)}{2 v}\right]^{1 / 2} } \\
& +\left[\left\|v_{0}^{\prime \prime}\right\|^{2}+\frac{K\left(\left\|u_{0}\right\|^{2}+\left\|\sqrt{m_{0}} v_{0}\right\|^{2}\right)}{v}\right]^{1 / 2} .
\end{aligned}
$$

Hereafter all the norms $\|\cdot\|$ are in $L^{2}(\Omega)$.

In order to prove the exponential decay of solutions, we assume that

$$
\alpha>3 K \int_{\Omega} m_{0}(x) d x \text { and } v>K \int_{\Omega} m_{0}(x) d x .
$$

Main results of this paper are the following. 
Theorem 1. Let $\Omega=(0,1), 0<\mu<\min \{\alpha, v\}, K>0, u_{0} \in H^{2}(\Omega)$, $v_{0} \in H^{2}(\Omega)$ and $m_{0} \in H^{1}(\Omega)$. If $\lambda<K / 7$, then for all $T>0$ the problem (2.1)-(2.5) has a unique strong solution:

$$
\begin{aligned}
& u \in L^{\infty}\left(0, T ; H^{2}(\Omega)\right) \cap L^{2}\left(0, T ; H^{4}(\Omega)\right), \\
& u_{t} \in L^{2}\left(0, T ; L^{2}(\Omega)\right), \\
& v \in L^{\infty}\left(0, T ; H^{2}(\Omega)\right), \\
& v_{t} \in L^{\infty}\left(0, T ; H^{1}(\Omega)\right), \\
& m \in L_{l o c}^{\infty}\left(0, T ; H^{1}(\Omega)\right) \cap L^{\infty}\left(0, T ; L^{1}(\Omega)\right), \\
& m \geq 0, \\
& m_{t} \in L_{l o c}^{\infty}\left(0, T ; L^{2}(\Omega)\right) .
\end{aligned}
$$

If conditions (2.7) hold, then there exists a real $\theta>0$ such that

$$
\|u\|^{2}(t)+\|\sqrt{m} v\|^{2}(t) \leq\left[\left\|u_{0}\right\|^{2}+\left\|\sqrt{m_{0}} v_{0}\right\|^{2}\right] e^{-\theta t} .
$$

Theorem 2. Let $\Omega=\mathbb{R}, 0<\mu<\min \{\alpha, v\}, K>0, u_{0} \in H^{2}(\Omega), v_{0} \in$ $H^{2}(\Omega)$ and $m_{0} \in H^{1}(\Omega) \cap L^{1}(\Omega)$. If $\lambda<K / 7$, then the Cauchy problem (2.1)(2.4) has a unique strong solution satisfying (2.8) for all $T>0$. If (2.7) are valid, then (2.9) holds.

Proof. We prove these theorems simultaneously by the following way. First, exploiting the method of successive approximations, we construct a local solution. Then, using a priori estimates and small (in norms (2.6)) initial data, we extend the local solution to the whole interval $(0, T)$ with arbitrary $T>0$. Finally, we prove the exponential decay of solutions as $t \rightarrow \infty$.

\section{Preliminary results}

Lemma 1. For any $w_{0}(x) \in H^{2}(\Omega)$ and $f(x, t) \in L^{2}\left(0, T ; H^{2}(\Omega)\right)$ there is a $t_{1}>0$ such that there exists a unique solution $w \in L^{\infty}\left(0, t_{1} ; H^{2}(\Omega)\right)$ to the problem

$$
\begin{aligned}
& w_{t}+w w_{x}=K(f-w), \\
& w(x, 0)=w_{0}(x), \\
& w(0, t)=w(1, t)=0, \quad \text { if } \Omega=(0,1)
\end{aligned}
$$


which satisfies the inequality

$$
\|w\|_{H^{2}(\Omega)}^{2}(t) \leq C_{v}\left(\left\|w_{0}\right\|_{H^{2}(\Omega)}^{2}+K \int_{0}^{t}\|f\|_{H^{2}(\Omega)}^{2}(\tau) d \tau\right), t \in\left(0, t_{1}\right) .
$$

Proof. The proof can be found in [8], p. 954.

Lemma 2. For any $p_{0}(x) \in H^{2}(\Omega)$ and $f(x, t) \in L^{2}(Q)$, there exists a unique solution to the problem

$$
\begin{aligned}
& p_{t}+p p_{x}+\mu p_{x x}+v p_{x x x x}+\alpha p=f(x, t), \\
& p(x, 0)=p_{0}(x), \\
& p(-L, t)=p(L, t)=p_{x x}(-L, t)=p_{x x}(L, t)=0 \quad \text { if } \Omega=(-L, L)
\end{aligned}
$$

such that for all $t \in(0, T)$

$$
\begin{aligned}
& \|p\|_{H^{2}(\Omega)}^{2}(t)+\int_{0}^{t}\|p\|_{H^{4}(\Omega)}^{2}(\tau) d \tau \\
& \quad \leq C_{u}\left(\left\|p_{0}\right\|_{H^{2}(\Omega)}^{2}+\int_{0}^{t}\|f\|_{L^{2}(\Omega)}^{2}(\tau) d \tau\right) .
\end{aligned}
$$

Proof. The proof can be found in [15]. Estimate (3.5) does not depend on $L$. This allows to consider the problem (3.2)-(3.4) on any (-L, L), $L>0$ and then, passing to the limit as $L \rightarrow \infty$, to obtain a solution to the Cauchy problem (3.2), (3.3) (see, for instance, [16]).

\section{Lemma 3. For any}

$$
\begin{aligned}
& a(x, t) \in L^{\infty}\left(0, T ; H^{2}(\Omega) \cap H_{0}^{1}(\Omega)\right), \\
& b(x, t) \in L^{\infty}\left(0, T ; H^{1}(\Omega)\right), \\
& f(x, t) \in L^{2}\left(0, T ; H^{1}(\Omega)\right), \\
& q_{0}(x) \in H^{1}(\Omega)
\end{aligned}
$$

there exists a unique solution

$$
q(x, t) \in L^{\infty}\left(0, T ; H^{1}(\Omega)\right)
$$


to the following problem:

$$
\begin{aligned}
& q_{t}+a q_{x}+b q=f, \\
& q(x, 0)=q_{0}(x), \\
& q(0, t)=q(1, t)=0 \quad \text { if } \quad \Omega=(0,1) .
\end{aligned}
$$

Proof. In fact, multiplying (3.7) by $q$, integrating over $Q_{t}=\Omega \times(0, t)$ and using Gronwall's lemma, we obtain

$$
\|q\|^{2}(t) \leq\left(\left\|q_{0}\right\|^{2}+\|f\|_{L^{2}(Q)}^{2}\right) e^{C_{1} T}, t \in(0, T),
$$

where $C_{1}$ is a positive constant.

Differentiating (3.7) with respect to $x$, multiplying by $q_{x}$ and integrating over $\Omega$, we have

$$
\begin{aligned}
\frac{1}{2} \frac{d}{d t} & \left\|q_{x}\right\|^{2}(t)+\int_{\Omega}\left(\frac{1}{2} a_{x} q_{x}^{2}+\frac{1}{2}\left(a q_{x}^{2}\right)_{x}+b_{x} q q_{x x}+b q_{x}^{2}\right) d x \\
& =\int_{\Omega} f_{x} q_{x} d x .
\end{aligned}
$$

Notice that

$$
\begin{aligned}
\left|\int_{\Omega} b_{x} q q_{x} d x\right| & \leq \sup _{\Omega}|q|(t)\left\|b_{x}\right\|(t)\left\|q_{x}\right\|(t) \\
& \leq C \sqrt{\|q\|^{2}+\left\|q_{x}\right\|^{2}} \cdot\left\|q_{x}\right\| \\
& \leq C\left(\|q\|^{2}+\left\|q_{x}\right\|^{2}\right),
\end{aligned}
$$

where the constant $C>0$ does not depend on $t$. Therefore, integrating (3.11) over $t \in(0, T)$ and using the Cauchy and Gronwall inequalities, we conclude that

$$
\left\|q_{x}\right\|^{2}(t) \leq\left(\left\|q_{0}^{\prime}\right\|^{2}+\left\|f_{x}\right\|_{L^{2}(Q)}^{2}\right) e^{C_{2} T}, \quad t \in(0, T) .
$$

Estimates (3.10) and (3.12) imply (3.6). 


\section{Local solution}

Let $u^{0}=0$. For $n \geq 1, n \in \mathbb{N}$, we define approximations $u^{n}$, $v^{n}$ and $m^{n}$ as solutions to the following problem:

$$
\left.\begin{array}{l}
u_{t}^{n}+u^{n} u_{x}^{n}+\mu u_{x x}^{n}+v u_{x x x x}^{n}+\alpha u^{n}=m^{n} K\left(v^{n}-u^{n-1}\right), \\
v_{t}^{n}+v^{n} v_{x}^{n}=K\left(u^{n-1}-v^{n}\right), \\
m_{t}^{n}+\left(m^{n} v^{n}\right)_{x}=0, \\
u^{n}(x, 0)=u_{0}(x), v^{n}(x, 0)=v_{0}(x), m^{n}(x, 0)=m_{0}(x), \\
u^{n}(0, t)=u_{x x}^{n}(0, t)=u^{n}(1, t)=u_{x x}^{n}(1, t)=0, \\
v^{n}(0, t)=v^{n}(1, t)=0,
\end{array}\right\} \text { if } \Omega=(0,1) .
$$

By Lemma 1 , we conclude that for any $v_{0} \in H^{2}(\Omega)$ there exists $t_{1}>0$ such that for all $0<t<t_{1}$ equation (4.2) with imposed initial and boundary (in the case of the mixed problem) conditions (4.4) and (4.5) has a unique solution such that

$$
\left\|v^{n}\right\|_{H^{2}(\Omega)}^{2}(t) \leq C_{v}\left(\left\|v_{0}\right\|_{H^{2}(\Omega)}^{2}+K \int_{0}^{t}\left\|u^{n-1}\right\|_{H^{2}(\Omega)}^{2}(\tau) d \tau\right),
$$

where the constant $C_{v}$ does not depend on $v^{n}$.

Approximations $m^{n}(x, t)$ for $t \in\left(0, t_{1}\right)$ can be found by the formula:

$$
m^{n}(x, t)=m_{0}\left(y^{n}(0 ; x, t)\right) \exp \left\{-\int_{0}^{t} \frac{\partial v^{n}}{\partial x}\left(y^{n}(\tau ; x, t), \tau\right) d \tau\right\} \geq 0,
$$

where $y^{n}(\tau ; x, t)$ is a solution to the Cauchy problem

$$
\frac{d y}{d \tau}=v^{n}(y, \tau) ; \quad y(t ; x, t)=x,
$$

defined for every $v^{n} \in L^{\infty}\left(0, t_{1} ; H^{2}(\Omega)\right)$.

Setting in (3.2) $f(x, t)=K m^{n}\left(v^{n}-u^{n-1}\right)$, and taking into account Lemma 2, (4.6) and (4.7), we conclude from (4.1) that for a.e. $t \in\left(0, t_{1}\right)$

$$
\begin{aligned}
& \left\|u^{n}\right\|_{H^{2}(\Omega)}^{2}(t)+\int_{0}^{t}\left\|u^{n}\right\|_{H^{4}(\Omega)}^{2}(\tau) d \tau \\
& \quad \leq C_{u}\left(\left\|u_{0}\right\|_{H^{2}(\Omega)}^{2}+\int_{0}^{t}\left\|K m^{n}\left(v^{n}-u^{n-1}\right)\right\|_{L^{2}(\Omega)}^{2}(\tau) d \tau\right),
\end{aligned}
$$


where the constant $C_{u}$ does not depend on $u^{n}$.

Thus, all the approximations $u^{n}, v^{n}$ and $m^{n}$ are defined on $\left(0, t_{1}\right)$ and (4.6)-(4.8) hold.

The next step is to show the uniform in $n \in \mathbb{N}$ boundness of approximations on some interval $\left(0, t_{2}\right)$. Inequality (4.8) implies

$$
\begin{gathered}
\left\|u^{n}\right\|_{H^{2}(\Omega)}^{2}(t) \leq C_{u}\left[\left\|u_{0}\right\|_{H^{2}(\Omega)}^{2}+\int_{0}^{t} \int_{\Omega} K^{2}\left(m^{n}\right)^{2}\left|v^{n}-u^{n-1}\right|^{2} d x d \tau\right] \\
\leq C_{u}\left[\left\|u_{0}\right\|_{H^{2}(\Omega)}^{2}+2 K^{2} \int_{0}^{t} \sup _{\Omega}\left|m^{n}\right|^{2}\left(\left\|v^{n}\right\|^{2}+\left\|u^{n-1}\right\|^{2}\right)(\tau) d \tau\right] \\
\leq C_{u}\left[2 K^{2} \int_{0}^{t} A^{n-1}(\tau) B^{n-1}(\tau) d \tau+\left\|u_{0}\right\|_{H^{2}(\Omega)}^{2}\right], \quad t \in\left(0, t_{1}\right),
\end{gathered}
$$

where

$$
\begin{aligned}
A^{n-1}(\tau) & =\left\|m_{0}\right\|_{H^{1}(\Omega)}^{2} \exp \left\{2 C _ { v } \int _ { 0 } ^ { \tau } \left(\left\|v_{0}\right\|_{H^{2}(\Omega)}^{2}\right.\right. \\
+ & \left.\left.K \int_{0}^{s}\left\|u^{n-1}\right\|_{H^{2}(\Omega)}^{2} d \xi\right)^{1 / 2} d s\right\}
\end{aligned}
$$

and

$$
B^{n-1}(\tau)=\left\|v_{0}\right\|^{2}+K \int_{0}^{\tau}\left\|u^{n-1}\right\|^{2}(s) d s+\left\|u^{n-1}\right\|^{2}(\tau) .
$$

We remind that $u^{0}=0$. Hence, for arbitrary $\mathcal{R}>2 C_{u}\left\|u_{0}\right\|_{H^{2}(\Omega)}^{2}$, it holds $u^{0}<\mathcal{R}$. The inductive hypothesis

$$
\sup _{0 \leq \tau \leq t_{1}}\left\|u^{n-1}\right\|_{H^{2}(\Omega)}^{2}(\tau)<\mathcal{R}
$$

implies, by (4.9), that

$$
\begin{aligned}
& \sup _{0 \leq \tau \leq t_{1}}\left\|u^{n}\right\|_{H^{2}(\Omega)}^{2}(\tau) \leq C_{u}\left[\left\|u_{0}\right\|_{H^{2}(\Omega)}^{2}\right. \\
& \left.+2 K^{2} \int_{0}^{t_{1}}\left\|m_{0}\right\|_{H^{1}(\Omega)}^{2} e^{2 C_{v} \tau} \sqrt{\left\|v_{0}\right\|_{H^{2}(\Omega)}^{2}+K \mathcal{R} \tau}\left(\left\|v_{0}\right\|_{H^{2}(\Omega)}^{2}+K \mathcal{R} \tau+\mathcal{R}\right)(\tau) d \tau\right] .
\end{aligned}
$$

Consequently, there exists a real $t_{2}>0$ such that for all $n \in \mathbb{N}$

$$
\sup _{0 \leq \tau \leq t_{2}}\left\|u^{n}\right\|_{H^{2}(\Omega)}^{2}(\tau) \leq 2 C_{u}\left\|u_{0}\right\|_{H^{2}(\Omega)}^{2}<\mathcal{R}
$$


and estimates (4.6) and (4.7) imply that $v^{n}$ and $m^{n}$ are bounded uniformly in $n$ on $\left(0, t_{3}\right)$.

Now we prove the convergence of approximations. The functions

$$
U^{n}=u^{n}-u^{n-1}, \quad V^{n}=v^{n}-v^{n-1} \quad \text { and } \quad M^{n}=m^{n}-m^{n-1}
$$

satisfy the following problem:

$$
\begin{aligned}
& U_{t}^{n}+u_{x}^{n} U^{n}+u^{n-1} U_{x}^{n}+\mu U_{x x}^{n}+v U_{x x x x}^{n}+\alpha U^{n} \\
& =K\left(m^{n} V^{n}+v^{n-1} M^{n}-u^{n-1} M^{n}-m^{n-1} U^{n-1}\right), \\
& V_{t}^{n}+v^{n} V_{x}^{n}+v_{x}^{n-1} V^{n}=K\left(U^{n-1}-V^{n}\right), \\
& M_{t}^{n}+\left(v^{n} M^{n}+m^{n-1} V^{n}\right)_{x}=0, \\
& U^{n}(x, 0)=V^{n}(x, 0)=M^{n}(x, 0)=0, \\
& \left.\begin{array}{l}
U^{n}(0, t)=U_{x x}^{n}(0, t)=U^{n}(1, t)=U_{x x}^{n}(1, t)=0, \\
V^{n}(0, t)=V^{n}(1, t)=0,
\end{array}\right\} \text { if } \Omega=(0,1) .
\end{aligned}
$$

Considering (4.11) and (4.12) as equations of the type of (3.7) with $f$ depending on $U^{n-1}$, we obtain from (4.10) by (3.5) that there exist $t_{3}>0$ and $0<\beta<1$ such that

$$
\sup _{0 \leq t \leq t_{3}}\left\|U^{n}\right\|_{H^{2}(\Omega)}(t) \leq \beta \sup _{0 \leq t \leq t_{3}}\left\|U^{n-1}\right\|_{H^{2}(\Omega)}(t) .
$$

Let $t_{0}=\min \left(t_{i}\right), i=1,2,3$. Then a subsequence of $\left\{u^{n}\right\}$ converges in $L^{\infty}\left(0, t_{0} ; H^{2}(\Omega)\right)$ that implies the convergence of $\left\{v^{n}\right\}$ and $\left\{m^{n}\right\}$ in classes $L^{\infty}\left(0, t_{0} ; H^{2}(\Omega)\right)$ and in $L^{\infty}\left(0, t_{0} ; H^{1}(\Omega)\right)$ correspondingly.

The fact that the limit of the approximations is the required solution to (2.1)(2.5) is established by the usual way [16].

Lemma 4. A solution of (2.1)-(2.5), satisfying (2.8), is uniquely defined.

Proof. To prove uniqueness of the solution obtained, we consider two solutions $(u, v, m)$ and $(\tilde{u}, \widetilde{v}, \tilde{m})$ of (2.1)-(2.5). The functions $U=u-\widetilde{u}, V=v-\widetilde{v}$ 
and $M=m-\tilde{m}$ satisfy the following problem:

$$
\begin{aligned}
& U_{t}+u U_{x}+\widetilde{u}_{x} U+\mu U_{x x}+v U_{x x x x}+\alpha U \\
& =K[m(V-U)+M(\tilde{v}-\tilde{u})], \\
& V_{t}+v V_{x}+\widetilde{v}_{x} V=K(U-V), \\
& M_{t}+v M_{x}+\widetilde{m}_{x} V+m V_{x}+\widetilde{v}_{x} M=0, \\
& U(x, 0)=V(x, 0)=M(x, 0)=0, \\
& \left.\begin{array}{l}
U(0, t)=U_{x x}(0, t)=U(1, t)=U_{x x}(1, t)=0, \\
V(0, t)=V(1, t)=0,
\end{array}\right\} \text { if } \Omega=(0,1) .
\end{aligned}
$$

It is easy to see that equations (4.16) and (4.17) are of the type of (3.7) with $f$ depending on $U$. Therefore, applying (3.5) to (4.15), we get

$$
\|U\|_{H^{2}(\Omega)}^{2}(t) \leq C K \int_{0}^{t}\|U\|_{H^{2}(\Omega)}^{2}(\tau) d \tau .
$$

This implies that $U=0$ and, consequently, $V=0$ and $M=0$.

\section{Global solution}

We need global a priori estimates to extend the local solution to the whole interval $(0, T)$. First, we define the energy function

$$
E(t)=\|u\|^{2}(t)+\|\sqrt{m} v\|^{2}(t) .
$$

Then, multiplying (2.1) by $u,(2.2)$ by $m v$, summing up the results, integrating over $Q_{t}$ and taking into account (2.3), we obtain the first estimate:

$$
\begin{aligned}
E(t) & +\int_{0}^{t}\left[v\left\|u_{x x}\right\|^{2}(\tau)+\alpha\|u\|^{2}(\tau)+2 K\|\sqrt{m}(u-v)\|^{2}(\tau)\right] d \tau \\
& \leq E(0) .
\end{aligned}
$$

Multiplying (2.2) by $v$ and integrating over $Q_{t}$, we get

$$
\|v\|^{2}(t)+K \int_{0}^{t}\|v\|^{2}(\tau) d \tau \leq\left\|v_{0}\right\|^{2}+K \int_{0}^{t}\|u\|^{2}(\tau) d \tau .
$$


Two times successively differentiating (2.2) with respect to $x$, multiplying by $v_{x}$ and $v_{x x}$ respectively and integrating over $Q_{t}$, we come to the inequalities

$$
\begin{aligned}
\left\|v_{x}\right\|^{2}(t) & +\int_{0}^{t}\left(K-\sup _{\Omega}\left|v_{x}\right|\right)\left\|v_{x}\right\|^{2}(\tau) d \tau \\
& \leq\left\|v_{0}^{\prime}\right\|^{2}+K \int_{0}^{t}\left\|u_{x}\right\|^{2}(\tau) d \tau \\
& \leq\left\|v_{0}^{\prime}\right\|^{2}+\frac{K}{2} \int_{0}^{t}\left(\|u\|^{2}+\left\|u_{x x}\right\|^{2}\right)(\tau) d \tau
\end{aligned}
$$

and

$$
\begin{aligned}
\left\|v_{x x}\right\|^{2}(t) & +\int_{0}^{t}\left(K-7 \sup _{\Omega}\left|v_{x}\right|\right)\left\|v_{x x}\right\|^{2}(\tau) d \tau \\
& \leq\left\|v_{0}^{\prime \prime}\right\|^{2}+K \int_{0}^{t}\left\|u_{x x}\right\|^{2}(\tau) d \tau .
\end{aligned}
$$

Lemma 5. If $\lambda<K / 7$, then

$$
\sup _{\Omega}\left|v_{x}(x, t)\right|<K / 7 \quad \text { for all } t>0 .
$$

Proof. Indeed, when $t=0, \sup _{\Omega}\left|v_{x}\right| \leq\left\|v_{0}^{\prime}\right\|+\left\|v_{0}^{\prime \prime}\right\| \leq \lambda<K / 7$. Suppose that $\sup _{\Omega}\left|v_{x}\left(x, t_{*}\right)\right|=K / 7$ for some $t=t_{*}>0$. Then (5.1)-(5.4) give

$$
\begin{aligned}
K / 7 & =\sup _{\Omega}\left|v_{x}\right|\left(t_{*}\right) \leq\left\|v_{x}\right\|\left(t_{*}\right)+\left\|v_{x x}\right\|\left(t_{*}\right) \\
& \leq \sqrt{\left\|v_{0}^{\prime}\right\|^{2}+K E(0) / 2 \alpha+K E(0) / 2 v}+\sqrt{\left\|v_{0}^{\prime \prime}\right\|^{2}+K E(0) / v} \\
& =\lambda<K / 7 .
\end{aligned}
$$

This contradiction proves Lemma 5.

It follows from (2.3) that the concentration $m(x, t)$ satisfies the conservation law

$$
\int_{\Omega} m(x, t) d x=\int_{\Omega} m_{0}(x) d x=C \text { for all } t>0 .
$$

Moreover, (4.7) implies that $m(x, t) \geq 0$. 
Thus, if $\lambda<K / 7$, then

$$
\begin{aligned}
& u \in L^{\infty}\left(0, T ; L^{2}(\Omega)\right) \cap L^{2}\left(0, T ; H^{4}(\Omega)\right), \\
& u_{t} \in L^{2}\left(0, T ; L^{2}(\Omega)\right), \\
& v \in L^{\infty}\left(0, T ; H^{2}(\Omega)\right), \\
& v_{t} \in L^{\infty}\left(0, T ; L^{1}(\Omega)\right) \cap L^{2}\left(0, T ; H^{1}(\Omega)\right), \\
& m \in L_{l o c}^{\infty}\left(0, T ; H^{1}(\Omega)\right) \cap L^{\infty}\left(0, T ; L^{1}(\Omega)\right), \\
& m \geq 0, \\
& m_{t} \in L_{l o c}^{\infty}\left(0, T ; L^{2}(\Omega)\right)
\end{aligned}
$$

and these inclusions do not depend on $T>0$. This allows us to extend the local solution for all $t>0$ (see [17] for details). Uniqueness of a global solution follows directly from Lemma 4.

\section{Stability}

Multiplying (2.1) by $u,(2.2)$ by $m v$, summing up the results, integrating over $\Omega$ and taking into account (2.3) and the definition of $E(t)$, we obtain

$$
\frac{d}{d t} E(t)+v\left\|u_{x x}\right\|^{2}(t)+\alpha\|u\|^{2}(t)+2 K\|\sqrt{m}(u-v)\|^{2}(t) \leq 0 .
$$

Then we estimate

$$
\begin{aligned}
I_{1} & :=2 K\|\sqrt{m}(u-v)\|^{2}(t)=2 K \int_{\Omega} m\left(u^{2}-2 u v+v^{2}\right) d x \\
& \geq 2 K \int_{\Omega}\left(m v^{2}-m v^{2} / 2-2 m u^{2}+m u^{2}\right) d x \\
& =2 K \int_{\Omega}\left(m v^{2} / 2-m u^{2}\right) d x
\end{aligned}
$$

and

$$
\begin{aligned}
I_{2} & :=2 K \int_{\Omega} m u^{2} d x \leq 2 K\|u\|_{L^{\infty}(\Omega)}^{2} \int_{\Omega} m d x \\
& \leq 2 K\left(\|u\|^{2}+\left\|u_{x}\right\|^{2}\right) \int_{\Omega} m_{0} d x \\
& \leq 2 K\left(\frac{3}{2}\|u\|^{2}+\frac{1}{2}\left\|u_{x x}\right\|^{2}\right) \int_{\Omega} m_{0} d x .
\end{aligned}
$$


Taking into account the estimates for $I_{1}, I_{2}$, we get from (6.1)

$$
\begin{aligned}
\frac{d}{d t} E(t) & +\left(\alpha-3 K \int_{\Omega} m_{0} d x\right)\|u\|^{2}(t) \\
& +\left(v-K \int_{\Omega} m_{0} d x\right)\left\|u_{x x}\right\|^{2}(t)+K\|\sqrt{m} v\|^{2}(t) \leq 0 .
\end{aligned}
$$

Making use of (2.7), one can see that for all $t>0$ there exists a constant $\theta>0$ such that

$$
E(t) \leq E(0) e^{-\theta t}
$$

This completes the proof of Theorems 1 and 2 .

\section{REFERENCES}

[1] P.G. Saffman, On the stability of laminar flow of a dusty gas, J. Fluid Mech., 13 (1) (1962), $120-128$.

[2] P.S. Crooke, On growth properties of solutions of the Saffman dusty gas model, Z. Angew. Math. Phys., 23 (1) (1972), 182-200.

[3] H.B. Stewart and B. Wendroff, Two-phase flow: models and methods, J. Comput. Phys., 56 (1984), 363-409.

[4] Kh.A. Rakhmatulin, The basic gas dynamics of interpenetrating motions of compressible media (in Russian), Prikl. Mat. Mekh., 20 (1956), 185-195.

[5] J.R. Kliegel and G.R. Nickerson, Flow of Gas-Particle Mixtures in Axially Symmetric Nozzles, In: Detonation and two-phase flow, Eds. S.S. Penner and F.A. Williams, Academic Press, New York, 173-194, (1962).

[6] R.I. Nigmatulin, Dynamics of multiphase media, (in Russian) Nauka, Moscow, 1987. American edition: Dynamics of Multiphase Media. Vol. 1, 2, Hemisphere Publishing Corporation, New York (1991).

[7] N.A. Larkin, A model system of equations of the mechanics of heterogeneous media (in Russian), Chisl. Metody Mekh. Sploshn. Sredy, 9 (7) (1978), 60-66.

[8] G.G. Doronin, Correctness of the Saffman model of a dust-laden gas with allowance for compressibility, Differ. Equ., 31 (6) (1995), 952-959.

[9] G.G. Doronin and N.A. Larkin, Cauchy Problems for Equations of Two-Phase Flow, In: Differential Equation Theory, Ed. A.M. Blokhin, Nova Science Publishers Inc, New York, 32-40, (1996).

[10] G.G. Doronin, On solvability of the Cauchy problem for the dusty gas equations, Comp. Appl. Math., 18 (2) (1999), 163-172. 
[11] Y. Kuramoto and T. Tsuzuki, On the formation of dissipative structures in reaction-diffusion systems, Prog. Theor. Phys., 54 (1975), 687-699.

[12] G.I. Sivashinsky, Nonlinear analysis of hydrodynamic instability in laminar flames, Acta Astronautica, 4 (1977), 1177-1206.

[13] H.A. Biagioni, J.L. Bona, R. Iorio Jr and M. Scialom, On the Korteveg-de-Vries-KuramotoSivashinsky equation, Adv. Diff. Eqs., 1 (1) (1996), 1-20.

[14] E. Tadmor, The well-posedness of the Kuramoto-Sivashinsky equation, SIAM J. Math. Anal., 17 (4) (1986), 884-893.

[15] A.T. Cousin and N.A. Larkin, Initial boundary value problem for the Kuramoto-Sivashinsky equation, Mat. Contemp., 18 (2000), 97-109.

[16] O.A. Ladyzhenskaya, N.N. Ural'tseva and V.A. Solonnikov, Linear and quasilinear equations of parabolic type (in Russian), Nauka, Moscow, 1967. American edition: AMS, Translations of mathematical monographs, 23, (1968).

[17] F.E. Browder, On non-linear wave equations, Math. Z., 80 (1962), 249-264.

[18] G.G. Doronin and N.A. Larkin, Kuramoto-Sivashinsky model for a dusty medium, Math. Methods Appl. Sci., 26 (3) (2003), 179-192.

[19] A.N. Kraiko, On correctness of the Cauchy problem for a two-fluids model of a gas flow containing particles, J. Appl. Math. Mech., 46 (1983), 327-333. 